\title{
Breakthrough Wave Detection in a 3D Computer Model of Atrial Endo-Epicardial Dissociation
}

\author{
Éric Irakoze ${ }^{1,2}$, Vincent Jacquemet ${ }^{1,2}$ \\ ${ }^{1}$ Université de Montréal, Montreal, Canada \\ ${ }^{2}$ Hôpital du Sacré-Cœur de Montréal
}

\begin{abstract}
Experimental and clinical mapping of atrial fibrillation has revealed the occurrence of breakthrough activation patterns. These focal waves have been associated with endo-epicardial (endo-epi) dissociation and three-dimensional anatomical structures. To assess breakthrough detection techniques in computer models of atrial fibrillation, we created a $3 D$ cubic-mesh atrial model with locally controllable endo-epi dissociation. In this model, epi and endo layers were electrically coupled only at randomly-distributed discrete connection sites. Eighteen endo-epi connection patterns were generated. Dedicated finite-difference numerical methods were developed to handle these discontinuities in conduction. These configurations were designed to generate breakthroughs at predictable locations. We developed a breakthrough detection algorithm based on full-resolution activation maps of both the epi- and endocardial surfaces. Wave tracking was used to calculate the lifespan of breakthroughs. Nonpropagating passive responses and breakthroughs with too short lifespan were eliminated. The approach was manually and automatically validated in 48 episodes of fibrillation in models with varying number of endo-epi connections.
\end{abstract}

\section{Introduction}

Breakthrough activation patterns have been observed in experimental mapping of atrial fibrillation [1]. Endoepicardial (endo-epi) dissociation has been hypothesized to promote the occurrence of these focal patterns by creating intramural three-dimensional pathways for reentry [2-5]. This mechanism has been found to contribute to the stability and complexity of atrial fibrillation [6].

The rate of breakthroughs observed in a given tissue area may be used to quantify three-dimensional reentries and to characterize the substrate. In a clinical mapping study in patients with persistent atrial fibrillation, de Groot et al. observed breakthrough rates of $2.5 / \mathrm{s}$ in a $1-\mathrm{cm}^{2}$ area in the left atrium, 2.7/s in the right atrium and 3.3/s in the pulmonary veins [1].

In a modeling study, severe epicardial fibrosis was associated with increased breakthrough rates $(45 / \mathrm{s}$ in the whole atrial epicardium vs 6.4/s in control) during simulated persistent atrial fibrillation [7]. In that study, breakthroughs were identified by tracking waves using membrane potential maps. Another approach for breakthrough detection that also applies to experimental data involves the analysis of phase maps [8]. Breakthrough rates may however depend on the spatial and temporal resolution of mapping.

To validate breakthrough detection methods, we developed an atrial model with endo-epi dissociation and discrete endo-epi connections. Locations of breakthrough sites are expected to coincide with these discrete connections which will facilitate validation. Criteria for eliminating breakthroughs that would not be observable at experimental mapping resolution will be investigated.

\section{Methods}

\subsection{Endo-epicardial dissociation in a cubic atrial model}

A cubic mesh of the atria (whose vertices form a set $\Omega$ ) with $280 \mu \mathrm{m}$ resolution was created based on previous works [9]. Endo- and epicardial layers were identified by solving a Laplace problem with boundary values +1 at the epicardial surface and -1 at the endocardial surface. The isosurface with isovalue 0 partitioned $\Omega$ into two disjoint subsets $\Omega_{\text {epi }}$ and $\Omega_{\text {endo }}$.

Introducing layer dissociation in a cubic mesh is challenging because of the stair-case nature of the surface representation and the need to properly handle no-flux boundary conditions in an anisotropic medium.

The finite difference method [10] was applied to compute the diffusion matrix $\mathbf{D}(\boldsymbol{\Omega}, \sigma)$ to discretize the monodomain equation on a mesh $\Omega$ of grid points with conductivity tensor $\sigma$. The matrices $\mathbf{D}\left(\boldsymbol{\Omega}_{\text {epi }}, \sigma\right)$ and $\mathbf{D}\left(\boldsymbol{\Omega}_{\text {endo }}, \sigma\right)$ were computed on the isolated epi- and endocardial layers 

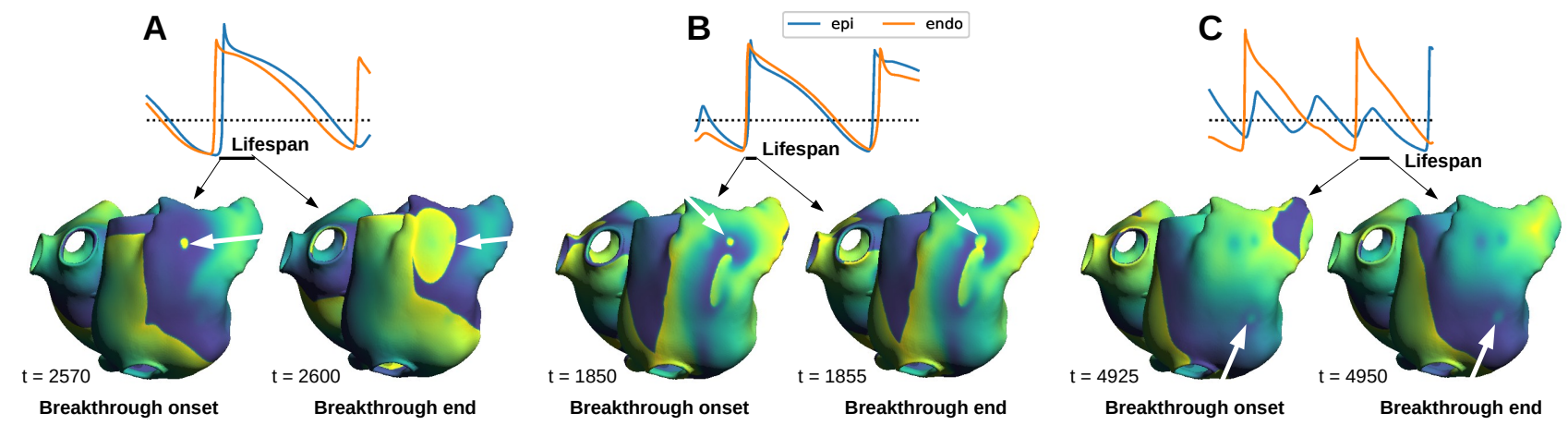

Figure 1. Examples of breakthrough detection: (A) valid breakthrough; (B) breakthrough with very short lifespan; (C) non-propagating breakthrough wave. Top: Membrane potential time course recorded at the endo- and epicardial surface near the breakthrough site. Bottom: color-coded membrane potential maps at the onset and the end of breakthrough waves.

and zero-padded so that they could be applied to vectors defined on $\Omega$. The diffusion matrix for partially dissociated layers was defined as

$$
\begin{aligned}
\mathbf{D}_{\text {dissoc }}(\boldsymbol{\Omega}, \sigma, \kappa)=\mathbf{D}(\boldsymbol{\Omega}, \kappa \sigma) & +\mathbf{D}\left(\boldsymbol{\Omega}_{\mathrm{epi}},(\mathbf{1}-\kappa) \sigma\right) \\
& +\mathbf{D}\left(\boldsymbol{\Omega}_{\mathrm{endo}},(\mathbf{1}-\kappa) \sigma\right)
\end{aligned}
$$

where $\kappa$ represents the coupling strength between the endo- and epicardial layers, $\kappa=1$ meaning fully coupled and $\kappa=0$ completely isolated. Heterogeneous dissociation can be specified when $\kappa$ is non-uniform. For any point $\mathbf{x}$ that has no nearest neighbor in the other layer, the value of $\kappa(\mathbf{x})$ does not affect the corresponding row of $\mathbf{D}(\boldsymbol{\Omega}, \sigma, \kappa)$. For points at the mid-myocardium boundary, however, the diffusion current is expressed as a linear combination of the fully coupled and the completely isolated cases. This operation preserves the conservation of current.

Inspired by Gharaviri's 2D model [4], discrete connections between the endo- and epicardial layers were introduced by setting $\kappa(\mathbf{x})$ to zero everywhere except within a given number $N_{c}$ of spheres of radius $2 \mathrm{~mm}$ and randomly placed over the mid-myocardium surface, where $\kappa(\mathbf{x})=1$. These connections provided discrete pathways for breakthrough patterns at predictable locations.

\subsection{Breakthrough detection}

Breakthrough detection during simulated atrial fibrillation was performed separately on the epi- and endocardial surfaces constructed from the set of square elements at the boundary of the cubic mesh. Depolarization and repolarization times were identified as the instant when membrane potential crosses a $-60 \mathrm{mV}$ threshold and stored in a file during the simulation. Surface waves were defined as connected regions with membrane potential above $-60 \mathrm{mV}$. Only connectivity through edges on the surface (and not within wall thickness) was considered. Connected com- ponents were computed using weighted quick-union data structure with path compression. Surface wave tracking was performed by finding the intersections between surface waves at times $t$ and $t+\Delta t$, where $\Delta t=1 \mathrm{~ms}$ [11]. Non-empty intersection was interpreted as propagation. A surface wave at time $t+\Delta t$ that had no intersection with any wave at time $t$ was considered to be a breakthrough wave. Breakthrough location and time were defined from the earliest activation site of that surface wave. Breakthrough waves were tracked until they merged with another wave or disappeared. This event determined the life span of the breakthrough wave. The size of the breakthrough wave (in number of vertices) at that moment was documented. During the lifespan of the breakthrough, the latest time of depolarization within the breakthrough wave was continuously updated. The output of the algorithm for each simulation was a list of breakthroughs with their five characteristics (location, onset time, lifespan, maximal size, latest depolarization time).

The list of breakthroughs was post-processed. The reason was that the lifetime of many breakthroughs was too short to be visually observable. A typical example was a breakthrough occurring $1 \mathrm{~ms}$ before the next wave front arrived. Also, some breakthroughs did not propagate and initiated a passive response instead. The criterion for counting a breakthrough was that the time interval between the earliest and latest activation time within the breakthrough wave was at least $10 \mathrm{~ms}$. This implied that their lifetime was $\geq 10 \mathrm{~ms}$. The breakthrough rate (BTR) was defined as the number of breakthrough waves per second satisfying that criterion.

\subsection{Simulation protocol}

Fibrillation was initiated in the isolated epicardial layer, as in previous works [4]. The description of membrane kinetics was also the same as [4]. Then, $N_{c}$ discrete endo-epi 
connections were introduced. We constructed 18 different models with $N_{c}$ ranging from 16 up to 256. In each model, 5 episodes of up to $5 \mathrm{~s}$ of fibrillation were simulated. The occurrence of self-termination within $5 \mathrm{~s}$ was documented.

Breakthroughs were automatically identified in all simulations. Detection was manually validated in a selected number of cases. Breakthrough rates in the epicardium and the endocardium were computed for three time windows: $0.3-1 \mathrm{~s}, 1-3 \mathrm{~s}$, and 3-5 s. For statistical analysis, simulations were grouped into four bins: $N_{c}<40$ (weak coupling, $n=15$ ), $40 \leq N_{c}<80$ (moderate coupling, $n=15$ ), $80 \leq N_{c}<160$ (stronger coupling, $n=35$ ), and $N_{c} \geq 160$ (strongest coupling, $n=25$ ).

\section{Results}

Simulated episodes of atrial fibrillation were sustained for more than $5 \mathrm{~s}$ in $100 \%$ of the cases when $N_{c}<40$, in $93 \%$ of the cases when $40 \leq N_{c}<80$, and in $54 \%$ of the cases when $80 \leq N_{c}<160$. Fibrillation always selfterminated when more than 160 connections were present. Breakthroughs were analyzed in sustained episodes only.

In all simulations, a total of 37,189 breakthroughs were detected in the epi and 89,184 in the endo. Among them, 5,265 or $14 \%$ (epi), and 15,055 or $17 \%$ (endo) satisfied the 10 -ms propagation criterion. Figure 1 shows examples of valid and excluded breakthroughs in the epicardium. The origin of most of them was located in the discrete endo-epi connections. The remaining breakthroughs, 6 in the epi and 185 the endo, were associated with three-dimensional structures such as interatrial connections and the pectinate muscles, as identified by manual validation.

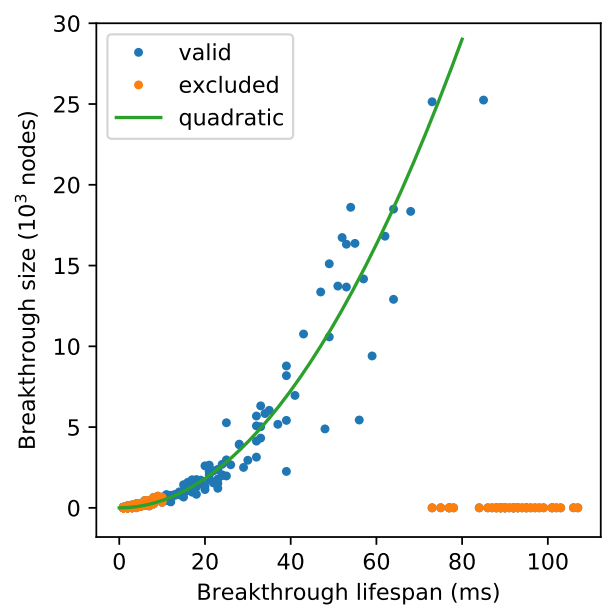

Figure 2. Characteristics of detected breakthroughs during a fibrillation episode. Excluded breakthroughs stopped propagating within $10 \mathrm{~ms}$ after breakthrough time. The solid line is of the form: size $=$ const $\times$ lifespan $^{2}$.
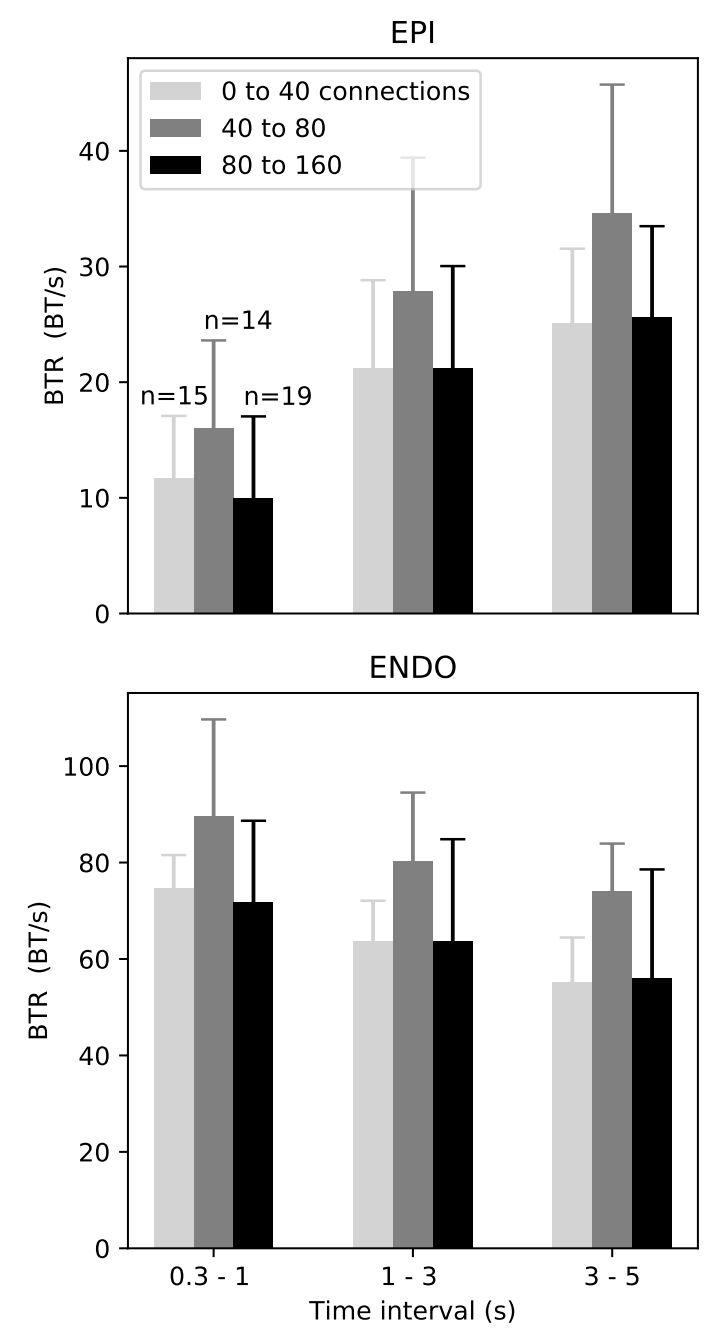

Figure 3. Breakthrough rate BTR (BT/s) in epi (top panel) and endo (bottom panel) during simulated fibrillation. Error bars represent standard deviation over fibrillation episodes. Bars are grouped by time intervals and are colored by the number of endo-epi connections.

The median lifespan of valid breakthroughs was $23 \mathrm{~ms}$ (quartiles: 20 and $26 \mathrm{~ms}$ ) in the epi and $94 \mathrm{~ms}$ (quartiles: 83 and $108 \mathrm{~ms}$ ) in the endo. The maximum size (area) of the breakthrough waves was approximately related to the square of their lifespan (Fig. 2), in agreement with a focal activation pattern. Data points excluded due to propagation failure were characterized by a small maximum size despite long-enough lifespan (Fig. 2).

The breakthrough rates in the epi- and in the endocardial surfaces were analyzed in three time intervals: the transient phase $(t<1 \mathrm{~s})$ where reentries migrated from the epi- to the endocardial layer, and then two intervals of two seconds. Mean and standard deviation of break- 
through rates during sustained fibrillation episodes are reported in Fig. 3. Breakthroughs were more frequent in the presence of a moderate number of connections and in the endocardial surface. The time evolution (increasing in the epi and decreasing in the endo) was caused by the initiation procedure that started fibrillation in the epicardium only.

Three-way analysis of variance (BTR as a function of number of connections, endo-epi layer and time intervals) confirmed that the effects of connections, layer and the interaction layer $\times$ time were all statistically significant $(p<0.001)$.

\section{Discussion}

The fibrillation model presented here extended to 3D the Gharaviri's 2D model and showed similar dynamics, notably larger breakthrough rates for intermediate endo-epi dissociation. Our emphasis was not on clinical applications but rather on testing a numerical method to introduce layer dissociation and on validating a breakthrough detection approach based on high-resolution simulated activation maps and wave tracking. The modified finite difference numerical methods may be used to simulate any spatially heterogeneous endo-epi coupling. Breakthroughs might then be observed anywhere rather that in discrete locations as in the present model (which was designed to identify possible false positives). The criterion of $10 \mathrm{~ms}$ propagation after breakthrough proved to be reliable for removing artifacts and identifying breakthroughs that might be observable at the lower resolutions used for experimental mapping. This would facilitate the comparison of breakthrough rates with clinical data.

\section{Conclusion}

Endo-epi dissociation combined with discrete coupling can be implemented in 3D cubic mesh models to create transmural reentries and breakthrough patterns. Such model may be used to validate mapping signal analysis software.

\section{Acknowledgments}

This work was supported by the Natural Sciences and Engineering Research Council of Canada (NSERC grant RGPIN-2020-05252).

\section{References}

[1] de Groot NM, Houben RP, Smeets JL, Boersma E, Schotten U, Schalij MJ, Crijns H, Allessie MA. Electropathological substrate of longstanding persistent atrial fibrillation in patients with structural heart disease. Circulation 2010; 122(17):1674-1682.
[2] Eckstein J, Zeemering S, Linz D, Maesen B, Verheule S, van Hunnik A, Crijns H, Allessie MA, Schotten U. Transmural conduction is the predominant mechanism of breakthrough during atrial fibrillation: evidence from simultaneous endo-epicardial high-density activation mapping. Circ Arrhythm Electrophysiol 2013;6:334-341.

[3] Verheule S, Tuyls E, Gharaviri A, Hulsmans S, van Hunnik A, Kuiper M, Serroyen J, Zeemering S, Kuijpers NHL, Schotten U. Loss of continuity in the thin epicardial layer because of endomysial fibrosis increases the complexity of atrial fibrillatory conduction. Circ Arrhythm Electrophysiol 2013;6:202-211.

[4] Gharaviri A, Verheule S, Eckstein J, Potse M, Kuijpers NHL, Schotten U. A computer model of endo-epicardial electrical dissociation and transmural conduction during atrial fibrillation. Europace 2012;14 Suppl 5:v10-v16.

[5] Hansen BJ, Zhao J, Csepe TA, Moore BT, Li N, Jayne LA, Kalyanasundaram A, Lim P, Bratasz A, Powell KA, Simonetti OP, Higgins RSD, Kilic A, Mohler PJ, Janssen PML, Weiss R, Hummel JD, Fedorov VV. Atrial fibrillation driven by micro-anatomic intramural re-entry revealed by simultaneous sub-epicardial and sub-endocardial optical mapping in explanted human hearts. Eur Heart J 2015;36(35):23902401.

[6] Eckstein J, Maesen B, Linz D, Zeemering S, van Hunnik A, Verheule S, Allessie M, Schotten U. Time course and mechanisms of endo-epicardial electrical dissociation during atrial fibrillation in the goat. Cardiovascr Res 2011; 89:816-824.

[7] Gharaviri A, Bidar E, Potse M, Zeemering S, Verheule S, Pezzuto S, Krause R, Maessen JG, Auricchio A, Schotten U. Epicardial fibrosis explains increased endo-epicardial dissociation and epicardial breakthroughs in human atrial fibrillation. Front Physiol 2020;11:68.

[8] Roney CH, Ng FS, Debney MT, Eichhorn C, Nachiappan A, Chowdhury RA, Qureshi NA, Cantwell CD, Tweedy JH, Niederer SA, Peters NS, Vigmond EJ. Determinants of new wavefront locations in cholinergic atrial fibrillation. Europace 11 2018;20(suppl 3):iii3-iii15.

[9] Irakoze É, Jacquemet V. Simulated P wave morphology in the presence of endo-epicardial activation delay. Europace 2018;20(suppl_3):iii16-iii25.

[10] Buzzard GT, Fox JJ, Siso-Nadal F. Sharp interface and voltage conservation in the phase field method: application to cardiac electrophysiology. SIAM J Sci Comput 2008; 30(2):837-854

[11] Gagné S, Jacquemet V. Time resolution for wavefront and phase singularity tracking using activation maps in cardiac propagation models. Chaos 2020;30(3):033132.

Address for correspondence:

Vincent Jacquemet

Hôpital du Sacré-Cœur de Montréal, Centre de Recherche

5400 boul. Gouin Ouest

Montreal (Québec) Canada H4J 1C5

vincent.jacquemeteumontreal.ca 